\title{
FLUX-LATTICE MELTING IN TWO-DIMENSIONAL DISORDERED SUPERCONDUCTORS
}

\author{
Mai Suan $\mathrm{Li}$ \\ Institute of Physics, Al. Lotnikow 32/46, 02-668 Warsaw, Poland \\ Thomas Nattermann \\ Institut für Theoretische Physik, Universität zu Köln, Zülpicher Str. 77, D-50937 Köln, Germany
}

(Dated: 17.1.03)

\begin{abstract}
The flux line lattice melting transition in two-dimensional pure and disordered superconductors is studied by a Monte Carlo simulation using the lowest Landau level approximation and quasiperiodic boundary condition on a plane. The position of the melting line was determined from the diffraction pattern of the superconducting order parameter. In the clean case we confirmed the results from earlier studies which show the existence of a quasi-long range ordered vortex lattice at low temperatures. Adding frozen disorder to the system the melting transition line is shifted to slightly lower fields. The correlations of the order parameter for translational long range order of the vortex positions seem to decay slightly faster than a power law (in agreement with the theory of Carpentier and Le Doussal) although a simple power law decay cannot be excluded. The corresponding positional glass correlation function decays as a power law establishing the existence of a quasi-long range ordered positional glass formed by the vortices. The correlation function characterizing a phase coherent vortex glass decays however exponentially ruling out the possible existence of a phase coherent vortex glass phase.
\end{abstract}

PACS numbers:

\section{INTRODUCTION}

The nature of the mixed phase in type-II twodimensional (2D) superconductors, despite considerable experimental, theoretical and numerical effort, remains unclear. In the mean field approximation the vortices of a pure system are known to form an Abrikosov lattice ${ }^{1}$. In $\mathrm{D}=3$ dimensions thermal fluctuations reduce the upper critical field line as first shown by Eilenberger ${ }^{2}$ leaving a vortex liquid phase between the transition line and the mean field $H_{c 2}(T)$. In $\mathrm{D}=2$ dimensions the phase diagram between the critical field lines $H_{c 1}(T)$ and $H_{c 2}(T)$ is still under debate. Applying the Kosterlitz-Thouless-Berezinskii-Halperin-NelsonYoung (KTBHNY) theory ${ }^{3.4 .5 .6 .7}$ originally developed for melting in $2 \mathrm{D}$ crystals to superconductors 8.9 one obtains a phase diagram with a solid, a hexatic and a liquid phase. The hexatic phase was observed in recent simulations 10 for the pinned XY model and low external magnetic fields. However, the possibilty that the transition is not of KTBHNY type was not excluded ${ }^{9}$. Indeed, for higher densities of dislocations (i.e. their core energy is low) a first order transition was found 11 . Moreover, vortices are not hard core particles. This applies in particular if they are described in the lowest Landau level(LLL) approximation where the density of vortices is high. With the help of a high temperature series expansion starting with the LLL approximation Hikami et $a^{112}$ found indeed a first order transition from the quasi-long range ordered vortex lattice to the liquid phase. In this case the intermediate hexatic phase disappears. The same result was obtained by Tesanovic and Xing 13 by Monte Carlo simulations using the LLL approximation ${ }^{14.15}$. However, theoretical works of Moore and coworkers ${ }^{16.17}$ raise doubts about the existence of the quasi-long range ordered vortex lattice and suggest that the flux liquid phase exists at any nonzero temperature. The simulations based on the LLL approximation gave conflicting results regarding the flux lattice melting in a clean 2D system. Using quasi-periodic boundary condition on a plane, $\mathrm{Hu}$ and MacDonald ${ }^{18}$, and Kato and Nagaosa ${ }^{19}$ have found a first order transition from a quasi-long range ordered vortex lattice phase to a vortex liquid. However, the quasi-solid phase was not detected by simulations on a sphere $\mathrm{2}^{20.21 .22}$.

In the presence of disorder the situation becomes even more complicated. It has long been predicted that in the presence of frozen-in disorder any long range crystalline order of a vortex lattice phase is destroyed in less than four dimensions ${ }^{23}$. Instead, a liquid-like phase was expected to occur leaving only short range crystalline order at length scales smaller than the Larkin length ${ }^{24}$. Recently, it was suggested that a Bragg glass phase with quasi-long range order can exist in three dimensional impure superconductors 25.26 .27 .28 provided the disorder is weak enough such that the dislocations cannot proliferate. Its existence in three dimensions was supported by further analytical 29.30 .31 and numerical ${ }^{32.33}$ studies. Experimental evidence for the existence of the Bragg glass phase was provided recently by neutron diffraction data 34 .

The situation in the $2 \mathrm{D}$ case is less clear. For clean systems the KTBHNY theory of melting predicts a dislocation driven melting transition from a positionally quasilong range ordered to a hexatic phase. The transition temperature is $T_{m}=\frac{a_{\triangle}^{2}}{4 \pi} \frac{\mu(\lambda+\mu)}{\lambda+2 \mu}$. Here $\lambda=c_{11}-2 c_{66}$ 
and $\mu=c_{66}$ denote the effective Lamé coefficients, $a_{\triangle}^{2}=\frac{2}{\sqrt{3}} \frac{\phi_{0}}{B}$ and $\phi_{0}=\frac{h c}{2 e}$ denotes the flux quantum. For $T<T_{m}$ the structure factor

$$
S(\mathbf{k}=\mathbf{G}+\mathbf{q}) \sim|\mathbf{q}|^{2-\eta_{\mathbf{G}}(T)}
$$

shows quasi-Bragg peaks with a temperature dependent exponent

$$
\eta_{\mathbf{G}}(T)=\frac{|\mathbf{G}|^{2} T}{4 \pi \mu} \frac{\lambda+3 \mu}{\lambda+2 \mu} .
$$

In the 2D Abrikosov triangular lattice where the screening length diverges with vanishing thickness of the film, $\lambda$ becomes scale dependent $\lambda(L) \sim L^{2}$, implying $\eta_{\mathbf{G}}\left(T_{m}\right)=$ $\frac{|\mathbf{G}|^{2} a_{\triangle}^{2}}{16 \pi^{2}}=\frac{1}{3} \quad\left(|\mathbf{G}|^{2}=\frac{16 \pi^{2}}{3 a_{\triangle}^{2}}\right)$. In finite systems of size $L_{s}$, $\eta_{\mathbf{G}}\left(T_{m}\right)$ may be larger because the effective value of $\lambda(L)$ is still finite. Above the melting transition the translational long range order of the lattice decays on scales $L>\xi \approx \exp \left[\frac{b T_{m}}{T-T_{m}}\right]^{\nu}$ with $\nu \approx 0.3696$ ․․ In finite systems one will see a slightly larger melting temperature defined by $L_{s} \approx \xi(T)$. The hexatic phase shows still quasi-long range order of the bond orientations which will disappear at a second transition at higher temperatures. As mentioned already, the possibility that the transition from the crystalline to the liquid phase is first order cannot be excluded.

Adding disorder to a purely elastic $2 \mathrm{D}$ system, i.e. if one suppresses dislocations by hand, there is at low temperatures a glassy phase with a structure factor

$$
S(\mathbf{k}=\mathbf{G}+\mathbf{q}) \sim|\mathbf{q}|^{2-\tilde{\eta}_{\mathbf{G}}(T) \ln \left(|\mathbf{q}| R_{a}\right)}
$$

where $\tilde{\eta}_{\mathbf{G}}(T) \sim\left(\frac{T-T_{g}}{T_{g}}\right)^{2}$ with $T_{g}$ being the glass temperature and $R_{a}$ is the length on which the vortex displacements become of the order of the lattice spacing $a_{\triangle}$. One should also note that $|\mathbf{q}|$ has to be smaller than $L_{c o}^{-1}$ where $L_{c o}$ is a cross-over length scale to the asymptotic behavior. The phase characterized by the structure (31) was found by Carpentier and Le Doussa ${ }^{35}$ following earlier work by Cardy and Ostlund 36 . We will call this phase therefore the Cardy-Ostlund-Carpentier-Le Doussal (COCD) phase. Contrary to the Bragg glass phase in $\mathrm{D}=3$ dimensions this phase does not show infinitely sharp Bragg peaks for $|\mathbf{q}| \rightarrow 0$ but the Bragg peaks saturate at $q R_{a} \lesssim e^{-2 / \tilde{\eta}_{G}}$ as can be seen from (3). Moreover, this phase is not stable with respect to dislocations which appear on a length scale $\frac{35.37 .38}{}$

$$
L_{d i s} \sim R_{a} e^{c_{1}\left[\frac{T_{m}-T}{T} \ln \left(R / R_{a}\right)\right]^{1 / 2}}
$$

due to the effect of the disorder. Here $c_{1}$ is a numerical constant. Since in the thermodynamic limit the glass temperature $T_{g}=\frac{3}{2 \pi} a_{\triangle}^{2} \frac{\mu(\lambda+2 \mu)}{(\lambda+3 \mu)}$ is much higher than the melting temperature $T_{m}\left(\left.\frac{T_{g}}{T_{m}}\right|_{\tilde{\lambda}_{e f f} \rightarrow \infty}=6\right)$ one will never observe the glass transition. Thus, for system sizes $L_{s}<$ $L_{d i s}$ we have to expect to see essentially a temperature driven melting transition from a low- $T$ COCD phase to a vortex liquid.

It should be noted that the LLL simulations ${ }^{39}$ for disordered systems fail to see any evidence for the existence of the COCD phase in even weakly disordered $2 \mathrm{D}$ superconductors of spherical geometry. The same problem but for a plane with the quasi-periodic boundary condition has not been studied yet.

In this paper we employ the LLL approximation and quasi-periodic boundary condition on a plane to study disordered 2D type-II superconductors by Monte Carlo (MC) simulations using the standard Ginzburg-Landau model where the impurities are introduced via fluctuations of the local critical temperature. The disorder is assumed to be weak such that the distance $L_{d i s}$ between dislocations is much larger than system sizes studied and hence the dislocations can be excluded.

In order to study the glass phase we monitor the Fourier transform of the density-density correlation function ${ }^{18,19}$

$$
\chi_{D D}(\mathbf{k})=\int_{\mathbf{r}} \int_{\mathbf{r}^{\prime}} \overline{<|\Psi(\mathbf{r})|^{2}\left|\Psi\left(\mathbf{r}^{\prime}\right)\right|^{2}>} e^{i \mathbf{k} \cdot\left(\mathbf{r}-\mathbf{r}^{\prime}\right)},
$$

where $\Psi(\mathbf{r})=|\Psi| e^{i \varphi}$ denotes the superconducting order parameter and the overbar the disorder averaging, respectively. The value of this function at $\mathbf{k}=\mathbf{G}$ with $\mathbf{G}$ being a reciprocal lattice vector defines the intensity of Bragg peaks. A closely related correlation function is that of the order parameter for translational long range order defined as follows 37

$$
S(\mathbf{G}, \mathbf{r})=\overline{\langle\exp (i \mathbf{G} \cdot[\mathbf{u}(\mathbf{r})-\mathbf{u}(0)])>} .
$$

Here $\mathbf{u}(\mathbf{r})$ denotes the displacement field of the vortex positions. The latter follow from the condition $\Psi(\mathbf{r})=$ 0 . It is this correlation function which exhibits quasilong range order in the Bragg glass phase. The structure factor is the Fourier transform of $S(\mathbf{G}, \mathbf{r})$

$$
S(\mathbf{k})=\int d^{2} r e^{i \mathbf{k r}} S(\mathbf{G}, \mathbf{r})
$$

In analogy with spin glass theory ${ }^{40}$ one may further consider the positional glass correlation function ${ }^{37}$

$$
S_{P G}(\mathbf{G}, \mathbf{r})=\overline{|<\exp (i \mathbf{G} \cdot[\mathbf{u}(\mathbf{r})-\mathbf{u}(0)])>|^{2}}
$$

which may give signature of the existence of some residual 'glassy' order of the Abrikosov lattice. If $S_{P G}(\mathbf{G}, \mathbf{r})$ decays not faster than a power law for $|\mathbf{r}| \rightarrow \infty$ then a system is said to be in the positional vortex glass phase.

In analogy to the positional glass correlation function one can define the gauge-invariant phase-coherent vortex glass correlation function ${ }^{41} C_{V G}(\mathbf{r})$ :

$$
C_{V G}(\mathbf{r})=\overline{\left|<\Psi(\mathbf{r}) \Psi^{*}(0) e^{i\left(2 \pi / \Phi_{0}\right) \int_{\Gamma} d \mathbf{r} . \mathbf{A}}>\right|^{2}} .
$$

Note that $C_{V G}(\mathbf{r})$ itself depends on the path $\Gamma$ between $\mathbf{r}$ and 0 along which the vector potential is integrated ${ }^{37}$. To 
make this correlation function path independent and simultaneously gauge invariant, Moor $\frac{16}{\underline{16}}$ proposed to keep only the longitudinal component of the vector potential. This corresponds in the symmetric gauge to the complete neglect of the phase factor $\exp \left[i\left(2 \pi / \Phi_{0}\right) \int_{\Gamma} d \mathbf{r}\right.$.A $]$ in Eq. (7):

$$
C_{V G}(\mathbf{r})=\overline{\left|<\Psi(\mathbf{r}) \Psi^{*}(0)>\right|^{2}} .
$$

The correlations measured in (8) cannot be probed directly but have been proposed to characterize the glassy state of the vortex array. They are expected to be reflected however in the current voltage relation although a straightforward derivation of this connection is still missing 41 . The relation between the correlations measured by (51) and the dissipation due to flux creep is $\operatorname{closer}^{37}$. Since we do not discuss dynamical properties in this article we consider (8) and (5) (and (6) as two competing theoretical tools to characterize the glassy state of the vortex array. An exponential decay of (8) - as we find - would suggest, according to $\underline{41}$, the disappearence of the glassy state. This is in contrast to what one would conclude from the almost power-law like decay of (5) which suggest some remaining glassy COCD-behaviour in the low temperature state. Contrary to previous works we will focus on the behavior of correlation functions defined by Eqs. (5), (6) and (8).

In agreement with ref $\frac{18.19}{10}$ we find that the clean $2 \mathrm{D}$ system displays the quasi-long range ordered vortex lattice phase at low temperatures. This result is in variance with the result of Moore et $a^{20.21 .22}$ who found the vortex liquid phase at any temperature using the same model but with different geometry.

In the disordered case, contrary to Kienappel and Moore ${ }^{39}$, we showed that the glassy COCD phase exists at low temperatures. In this phase the translational invariance order parameter is found to decay as $S(\mathbf{G}, \mathbf{r}) \sim e^{-\tilde{\eta}_{\mathbf{G}} \ln ^{2} r}$ as predicted theoretically by Carpentier and Le Doussa ${ }^{35}$. However, due to small system sizes, the possibility that it decays as a simple power law is not excluded. The clarification of this point requires simulations for considerably larger system sizes. Our results indicate, however, that quasi-long range order of correlations measured by (6) exist at low temperatures.

In the glassy phase the phase coherent vortex glass order parameter decays as $C_{V G}(\mathbf{r}) \sim \exp \left(-r / R_{c}\right)$, where the correlation length $R_{c}$ depends not only on the temperature but also on the disorder. The stronger the disorder the larger is $R_{c}$ suggesting that the phase-coherent vortex glass ordering becomes more and more favourable as the disorder is enhanced.

The outline of the paper is as follows. In Sec. II we present the LLL approach in the Landau gauge. The transition from the vortex liquid to the ordered phase of the clean system is studied in Sec. III. The nature of ordering in the same system but this time with disorder is discussed in Sec. IV. The exponential decay of the phase coherent vortex glass is also presented in this sec- tion. Finally, in the last section we summarize our main results.

\section{MODEL}

Our simulation is based on the phenomenological Ginzburg-Landau model in the approximation of a uniform magnetic field $\mathbf{B}$. This is a reasonable approximation, since the effective screening length $\lambda_{\text {eff }}=2 \lambda_{L}^{2} / \mathrm{s}$ diverges for vanishing film thickness $s$. Here $\lambda_{L}$ denotes the bulk screening length. The quenched disorder is introduced via the fluctuations of the local phase transition temperature. The Ginzburg-Landau free energy of the superconductor is given by

$$
\begin{aligned}
F & =F_{c l}+F_{d i s}, \\
F_{c l} & =\int d^{2} r\left[\alpha(T)|\Psi|^{2}+\frac{\beta}{2}|\Psi|^{4}+\frac{1}{2 m}|\mathbf{D} \Psi|^{2}\right], \\
F_{d i s} & =\int d^{2} r \alpha_{0} \delta T_{c}(\mathbf{r})|\Psi(\mathbf{r})|^{2} .
\end{aligned}
$$

Here $\Psi$ is the complex order parameter representing the macroscopic wave function of the superconducting electrons. D denotes the gauge invariant derivative $\mathbf{D}=-i \hbar \nabla-\frac{2 e}{c} \mathbf{A}$ with $\mathbf{A}$ being the vector potential. $\mathbf{B}=\nabla \times \mathbf{A}, e$ and $m$ are the charge and mass of the electron, respectively. In the simulation we will use the the Landau gauge $\mathbf{A}=B(0, x, 0)$. We may go back to the symmetric gauge $\mathbf{A}=\frac{B}{2}(-y, x, 0)$ by a simple gauge transformation $\Psi_{L}=\Psi_{S} e^{i x y / l^{2}} . \alpha(T)=\alpha_{0}\left(T-T_{c}\right)$ and $\beta$ is a constant; $\alpha_{0}, \beta>0 . T_{c}$ denotes the zero-field meanfield transition temperature. $\delta T_{c}(\mathbf{r})$ is real and Gaussian distributed with

$$
\begin{aligned}
<\delta T_{c}(\mathbf{r})> & =0, \\
<\delta T_{c}(\mathbf{r}) \delta T_{c}\left(\mathbf{r}^{\prime}\right)> & =\zeta^{2} T_{c}^{2} \xi_{0}^{2} \delta_{\xi_{0}}\left(\mathbf{r}-\mathbf{r}^{\prime}\right) .
\end{aligned}
$$

$\delta_{\xi_{0}}(x)$ is a $\delta$-function of width of the order of the zero temperature correlation length $\xi_{0}=\hbar / \sqrt{2 m \alpha_{0} T_{c}}$. The typical fluctuations $\delta T_{c}(\mathbf{r})$ of the mean-field transition temperature are then of the order $\delta T_{c} \cong \zeta T_{c}$. It is convenient to collocate here the main characteristics of the model

$$
\begin{gathered}
B_{c 2}(0)=\frac{\alpha_{0} T_{c} m c}{\hbar e}, \quad b=\frac{B}{B_{c 2}(0)}, \quad G i=\left(\frac{m \beta}{2 \pi \hbar^{2} \alpha_{0}}\right)^{2}, \\
l=\sqrt{\frac{\hbar c}{2 e B}}=\frac{1}{\sqrt{b}} \xi_{0}=\frac{a_{0}}{\sqrt{2 \pi}}=\sqrt{\frac{\sqrt{3}}{4 \pi}} a_{\triangle} .
\end{gathered}
$$

Here $B_{c 2}(0)$ denotes the upper critical field at $T=0$ and $l$ the magnetic length. The density and the lattice constant of the flux line lattice are given by $\frac{1}{a_{0}^{2}}$ and $a_{\triangle}$, respectively. $G i$ is the Ginzburg number at $T=T_{c}$.

In the LLL approximation one expands the order parameter $\Psi$ in terms of eigenfunctions of the operator $\alpha+\mathbf{D}^{2} / 2 m$ of the lowest Landau level only. This restriction is a reasonable approximation provided that fluctuations in higher Landau level channels can be neglected. 
The precise range of applicability of the LLL approximation is still under debate ${ }^{20.42}$. However, one can expect that this approximation is valid for $B \gtrsim B_{c 2} / 13^{42}$. Since the basic length scale of the LLL approximation is given by the magnetic length $l=\sqrt{\hbar c / 2 e B}$ it is convenient to measure from now on all lengths in units of $l$, i.e. we replace everywhere $\frac{x}{l}$ by $x$ and $\frac{y}{l}$ by $y$.

We apply quasi-periodic boundary conditions to the order parameter inside a finite system with lengths $L_{x}$ and $L_{y}$ parallel to the $x$ - and $y$-direction, respectively. $\Psi\left(y+L_{y}, x\right)=\Psi(y, x)$ and $\Psi\left(y, x+L_{x}\right)=$ $\exp \left(-i L_{x} y / l\right) \Psi(y, x)$. Such boundary conditions are necessary to result in a phase change by $2 \pi N_{\phi}$ when orbiting the system. $N_{\phi}=\frac{L_{x} L_{y}}{2 \pi l^{2}}$ denotes the number of vortices in the system. We will denote $L_{x} / l=3^{1 / 4} \pi^{1 / 2} N_{x}$ and $L_{y} / l=2 \pi^{1 / 2} N_{y} / 3^{1 / 4}$, where $N_{x}$ and $N_{y}$ are the numbers of vortex columns and rows, respectively. The order parameter can then be written in the form 15

$$
\Psi(\mathbf{r})=\sqrt{\frac{\left|\alpha_{B}\right| l \pi^{\frac{1}{2}}}{\beta L_{y}}} \sum_{j=1}^{N_{\phi}} \sum_{s=-\infty}^{\infty} C_{j} e^{\frac{i y X_{j, s}-\left(x-X_{j, s}\right)^{2}}{2}} .
$$

In the last equation we have introduced the following notations

$$
\begin{aligned}
\alpha_{B} & =\alpha(T)+\hbar e B / m=\alpha_{0} T_{c}(1-b-t), \\
t & =\frac{T}{T_{c}}, X_{j, s}=j 2 \pi l / L_{y}+s L_{x} / l
\end{aligned}
$$

In the mean field theory the superconducting transition of clean systems occurs at $T_{c}(B)=T_{c}-\hbar e B / \alpha_{0} m c$ which is defined from the condition $\alpha_{B}=0 . X_{j, s}$ is the center of the cyclotron motion. The number of vortices $N_{\phi}$ must be chosen to be an integer, the coefficients $C_{j}$ are in general complex.

Using the expansion (12) we can rewrite $F_{c l}$ as follows

$$
\begin{array}{r}
\frac{F_{c l}}{\epsilon^{2} T}=\operatorname{sign}\left(\alpha_{B}\right) \sum_{j}\left|C_{j}\right|^{2}+\frac{3^{1 / 4}}{8 \sqrt{2} N_{y}} \sum_{n_{s}=-\infty}^{\infty} \sum_{n_{d}=-\infty}^{\infty}\left[f\left(n_{s}\right)\left|\exp \left[-\frac{\sqrt{3} \pi n_{d}^{2}}{N_{\phi}}\right] C_{\left[n_{s}+n_{d}\right]} C_{\left[n_{s}-n_{d}\right]}\right|^{2}+\right. \\
\left.+f\left(n_{s}+1 / 2\right)\left|\exp \left[-\frac{\sqrt{3} \pi\left(n_{d}-1 / 2\right)^{2}}{N_{\phi}}\right] C_{\left[n_{s}+n_{d}\right]} C_{\left[n_{s}-n_{d}+1\right]}\right|^{2}\right],
\end{array}
$$

where $C_{\left[n_{s}\right]}=C_{\bmod \left(n_{s}, N_{\phi}\right)}$. The only model dependent parameter of the model in the clean limit is $\epsilon$ given by

$$
\epsilon=\frac{\alpha_{B} \pi^{1 / 2} l}{\beta^{1 / 2} T^{1 / 2}}=\frac{1}{2 G i^{\frac{1}{4}}} \frac{(b+t-1)}{b^{1 / 2} t^{1 / 2}},
$$

which measures the distance to the mean-field phase boundary $\epsilon=0$. Here $b$ denotes the magnetic field in units of the mean-field upper critical field $B_{c 2}$ at $T=0$ (compare (11)). Below we will find the phase transitions at a critical value $\epsilon_{c}$ of $\epsilon$ which can be inverted into a band of transition lines $b_{c}(t)$ parameterized by the Ginzburg number of the corresponding superconductor:

$$
b_{c}(t ; G i, \zeta=0)=\tilde{b}_{c}\left(t, \epsilon_{c} G i^{\frac{1}{4}}\right) \equiv 1-t+\varkappa_{c}^{2} t-\varkappa_{c} \sqrt{2 t(1-t)+\varkappa_{c}^{2} t^{2}}, \varkappa_{c}=\sqrt{2} \epsilon_{c} G i^{\frac{1}{4}} .
$$

Finally,

$$
f\left(n_{s}\right)=\operatorname{Erf}\left(N_{x} 3^{1 / 4}(2 \pi)^{1 / 2}-n_{s} N_{y}^{-1} 3^{1 / 4}(2 \pi)^{1 / 2}\right)+\operatorname{Erf}\left(n_{s} N_{y}^{-1} 3^{1 / 4}(2 \pi)^{1 / 2}\right),
$$

where $\operatorname{Er} f(x)$ is an error function. It should be noted that different groups used different notations for the dimensionless parameter $\epsilon$. Our $\epsilon=t\left(\right.$ Ref. 18) $=t / 2($ Ref. 19) $)=\alpha_{T} / \sqrt{2}($ Ref. 12) $=g / \sqrt{2}($ Ref. 13) .

To express the disorder term $F_{d i s}$ in terms of the LLL coefficients $C_{j}$, we expand the random Gaussian disorder in renormalized Hermite polynomials $u_{k}(x)$ and harmonics

$$
\delta T_{c}(\mathbf{r})=\frac{\zeta T_{c} \xi_{0}}{\left(L_{y} l\right)^{1 / 2}} \sum_{k=0}^{\infty} \sum_{m=-\infty}^{\infty} a_{k m} e^{2 \pi i m y l / L_{y}} u_{k}(x), \quad u_{k}(x)=\left(2^{k} k ! \sqrt{\pi}\right)^{-1 / 2} e^{-x^{2} / 2} H_{k}(x) .
$$

Here $H_{k}(x)$ are Hermite polynomials and $a_{k m}$ are complex random numbers. To satisfy $\delta T_{c}(\mathbf{r})^{*}=\delta T_{c}(\mathbf{r})$ one has to choose $a_{k m}$ such that $a_{k,-m}=a_{k, m}$. Using the standard orthogonality relations one can show that $\delta T_{c}(\mathbf{r})$ satisfies 
Eq. (10) if $<a_{k m}>=0$ and $\left\langle a_{k m} a_{k^{\prime} m^{\prime}}^{*}>=\delta_{m m^{\prime}} \delta_{k k^{\prime}}\right.$. From Eqs. (12) and (18) we have

$$
\begin{array}{r}
\frac{F_{d i s}}{T}=\epsilon^{2} \tilde{\zeta}\left(\frac{l}{L_{y}}\right)^{1 / 2} \sum_{k=0}^{\infty} \sum_{n_{s}, n_{d}}\left(a_{k, 2 n d} C_{\left[n_{s}+n_{d}\right]} C_{\left[n_{s}-n_{d}\right]}^{*} L\left(k, n_{s}, n_{d}\right)+\right. \\
\left.a_{k, 2 n d-1} C_{\left[n_{s}+n_{d}\right]} C_{\left[n_{s}-n_{d}+1\right]}^{*} L\left(k, n_{s}+\frac{1}{2}, n_{d}-\frac{1}{2}\right)\right) .
\end{array}
$$

Here

$$
L\left(k, n_{s}, n_{d}\right)=e^{-\frac{\pi 3^{1 / 2}}{N_{y}^{2}} n_{d}^{2}} \int_{0}^{L_{x} / l} d x u_{k}(x) e^{-\left(x-\frac{3^{1 / 4} \pi^{1 / 2}}{N_{y}} n_{s}\right)^{2}}
$$

and the dimensionless parameter $\tilde{\zeta}$ which controls the relative disorder strength has the form

$$
\tilde{\zeta}=\frac{\zeta}{2 \pi^{1 / 2} G i^{1 / 4} \epsilon t^{1 / 2}}=\zeta \frac{b^{1 / 2}}{\pi^{1 / 2}(1-t-b)} .
$$

Substituting (12) into Eq. (44) one can express the Fourier transform of the density-density correlation function in the following form (again we measure $k^{-1}$ in units of $l$, i.e. we replace $k l$ by $k$ )

$$
\chi_{D D}(\mathbf{k})=\left(\frac{N_{\phi} \alpha_{B} \pi l^{2}}{\beta}\right)^{2} e^{-k^{2} / 2} \overline{<|\Delta(\mathbf{k})|^{2}>}
$$

where

$$
\Delta(\mathbf{k})=\sum_{j} C_{\left[j-n_{y}+N_{\phi}\right]}^{*} C_{j} e^{\frac{\pi l q_{x}}{L_{y}}\left(n_{y}-2 j\right)} .
$$

In what follow we will use $\overline{\left\langle|\Delta(\mathbf{k})|^{2}>\right.}$ to characterize the Bragg peaks.

Our MC simulations are performed for coefficients $C_{j} \in \mathbf{C}^{N_{\phi}}$ which are updated by the standard Metropolis algorithm. The candicate for the new configuration is generated by $C_{j} \rightarrow C_{j}+\epsilon \Delta C$ where $\Delta C$ is a complex number which is randomly chosen from the region $|\operatorname{Re} \Delta C| \leq 1$ and $|\operatorname{Im} \Delta C| \leq 1$ in the complex plane. $\epsilon$ is chosen to be of order 0.1 so that the acceptance ratio is $0.3 \sim 0.5$. The physical quantities are measured every $20 \sim 50 \mathrm{MC}$ steps.

\section{CLEAN SYSTEM}

The aim of this section is twofold. First, we want to check our code for the clean system $(\tilde{\zeta}=0)$ which was studied in detai ${ }^{18,19}$. Second, we consider the spatial behavior of the translational invariance correlation function which have not been studied previously by simulations.

The simulations were carried out for systems of five different sizes, each containing $4^{2}, 6^{2}, 8^{2}, 10^{2}$ and $12^{2}$ number of vortices. It took $3 \times 10^{4} \sim 10^{5} \mathrm{MC}$ steps to reach the thermal equilibration. The physical quantities were calculated over $10^{5} \sim 5 \times 10^{5}$ MC steps.

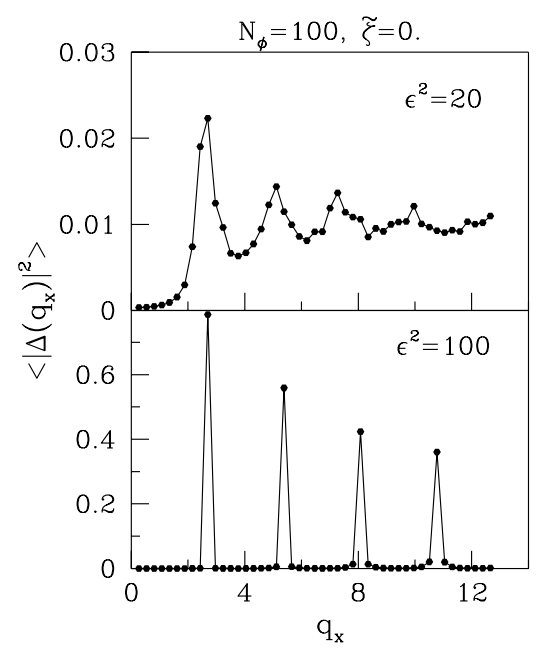

FIG. 1: The wave vector dependence of the structure factor for the clean system with $\tilde{\zeta}=0 . N_{\phi}=100, \epsilon^{2}=20$ and 100. $q_{x}$ is measured in units $\frac{1}{l}$.

Fig. 11 shows the temperature dependence of $<$ $\left|\Delta\left(q_{x}\right)\right|^{2}>=<\left|\Delta\left(q_{x}, 0\right)\right|^{2}>$ for $N_{\phi}=100, \epsilon^{2}=20$ and 100. At low temperatures (large $-\epsilon$ ) this quantity has the sharp peaks which are indicative for the existence of the quasi vortex lattice. At high temperatures the Bragg peaks are smeared out suggesting that we are in the vortex liquid phase.

In order to obtain the quasi solid-liquid transition temperature we monitor the scaling of the renormalized structure factor at the maximum position $q_{x}=G=$ $\frac{(4 \pi)^{1 / 2}}{3^{1 / 4}},<|\tilde{\Delta}(G)|^{2}>$, which is defined as follows

$$
<|\tilde{\Delta}(G)|^{2}>=N_{\phi}<|\Delta(G)|^{2}>/<|\Delta(0)|^{2}>.
$$

Fig. [2]shows the temperature dependence of $\left\langle|\tilde{\Delta}(G)|^{2}>\right.$ for various values of $N_{\phi}$. The transition temperature is defined from the point where the curves splay out and we find $\epsilon_{c}^{2}=43 \pm 2$. This can be compared with previous estimates $\epsilon_{c}^{2}=43.5 \pm 1.0$ by Hu and MacDonald $18, \epsilon_{c}^{2} \sim$ 50 by Tesanovic and Xing 13 , and $\epsilon_{c}^{2} \approx 49$ by Kato and Nagaosa ${ }^{19}$. The discrepancy between estimates of various groups is probably related to the fact that the scaling regime was not reached due to small system sizes used up to now. Since this regime, according to Kato and Nagaosa $\frac{19}{}$, corresponds to $N_{\phi}>36^{2}$ and the CPU time grows as $N_{\phi}^{5}$ it is beyond our computational facilities to reach it. 


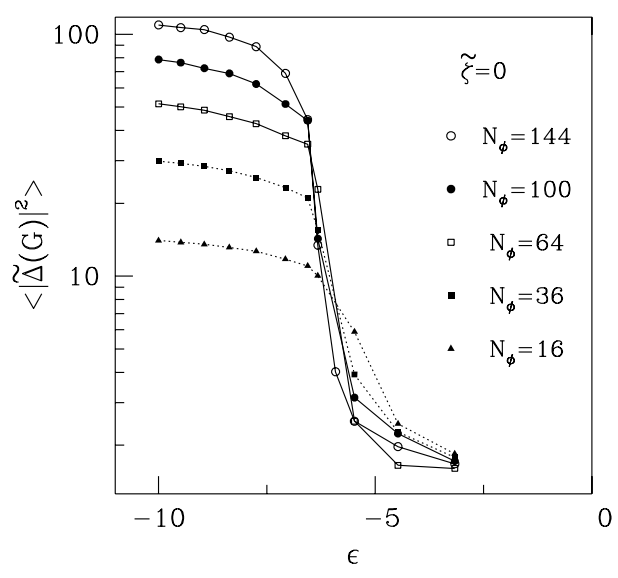

FIG. 2: The temperature and size dependence of the structure factor for the clean system. $N_{\phi}=16,36,64,100$ and 144 .

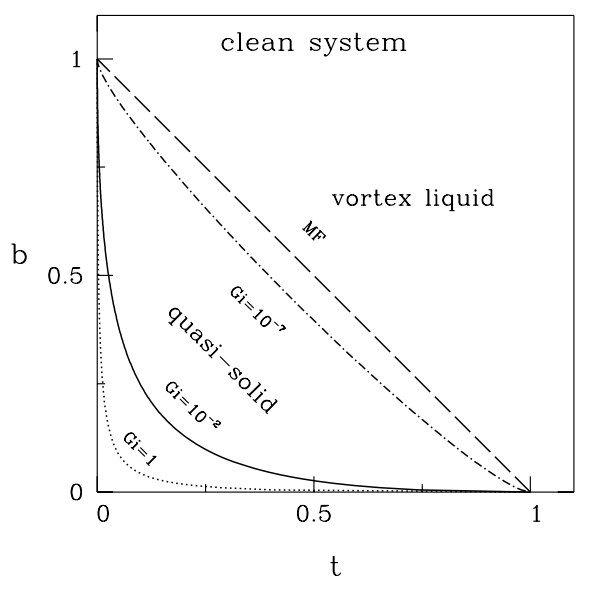

FIG. 3: The temperature-field phase diagram for the clean system. The dashed line denotes the mean field boundary between the vortex liquid and the quasi-solid phases. The dot-dashed, solid and dotted lines correspond to the phase boundary for Ginzburg number $G i=10^{-7}, 10^{-2}$ and 1 , respectively.

Fig. 3 shows the phase boundary between the vortex liquid and the quasi-solid phase using Eq. (16). We have chosen the Ginzburg number $G i=10^{-7}$ which is typical for low- $T_{c}$ superconductors, and $G i=10^{-2}$ and 1 for high- $T_{c}$ materials 43 . Since the thermal fluctuations enhance with $G i$, the quasi-long range ordered vortex lattice shrinks as one increases the Ginzburg number.

The distance dependence of the translational invariance order parameter $S(\mathbf{G}, \mathbf{r})$ for $N_{\phi}=144$ and for various values of $\epsilon$ is shown in Fig. 团 At low temperatures we find a power law behavior of the correlation function for translational long range order

$$
S(\mathbf{G}, \mathbf{r}) \sim r^{-\eta_{\mathbf{G}}(T)}
$$

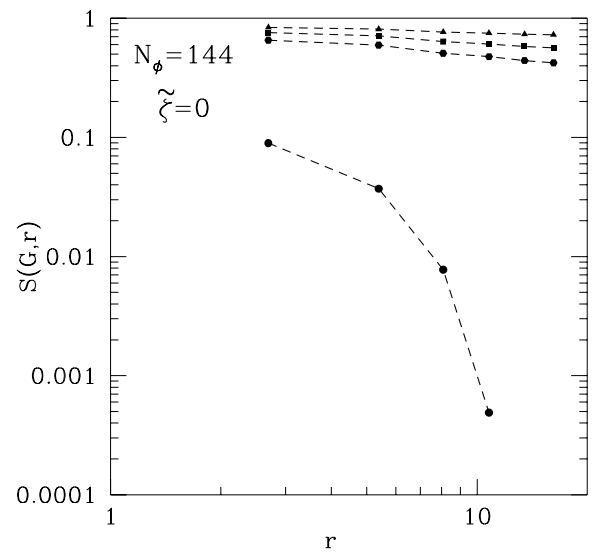

FIG. 4: The distance dependence of the translational invariance order parameter $S(\mathbf{G}, \mathbf{r})$ for the clean system. Closed circles, hexagons, squares and triangles correspond to $\epsilon^{2}=$ $10,50,70$ and 100, respectively. $r$ is measured in units of $l$.

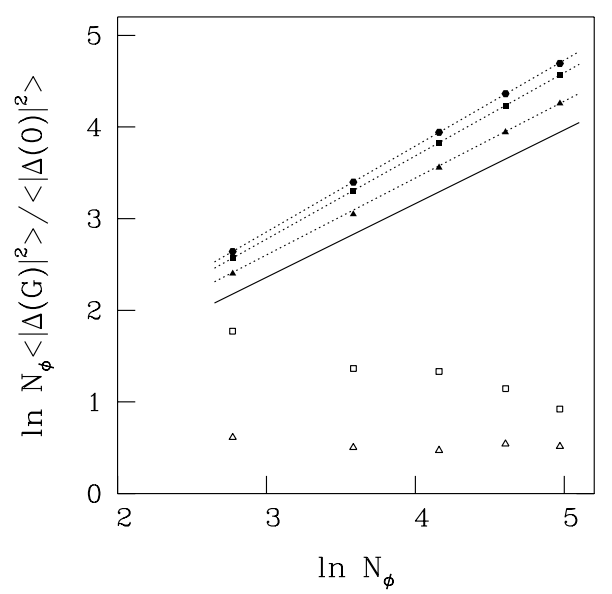

FIG. 5: Dependence of the structure factor on $N_{\phi}$ for the clean system. Open triangles, squares, and closed triangles, squares and hexagons correspond to $\epsilon^{2}=10,30,43,70$ and 100, respectively. Dotted lines are linear fits and the solid line corresponds to $\eta_{\mathbf{G}}=\frac{1}{3}$ from the KTBHNY theory.

whereas at high temperatures an exponential dependence on $r$ occurs.

One can define $\eta_{\mathbf{G}}$ from the distance dependence of $S(\mathbf{G}, \mathbf{r})$ shown in Fig. 4 However, in order to minimize finite size effects we calculate $\eta_{\mathbf{G}}$ from the dependence of $\left\langle|\tilde{\Delta}(G)|^{2}>\right.$ on $N_{\phi}$ in the quasi solid phase: $<|\tilde{\Delta}(G)|^{2}>\sim N_{\phi}^{1-\eta_{\mathbf{G}} / 2}$ (see Ref. 19). In the liquid phase $<|\tilde{\Delta}(G)|^{2}>$ does not depend on $N_{\phi}$. Fig. 5 plots $\ln <|\tilde{\Delta}(G)|^{2}>$ versus $\ln N_{\phi}$. The dependence of $\eta_{\mathbf{G}}(T)$ on parameter $\epsilon$ is presented in Fig. 6] At the melting temperature $\eta_{\mathbf{G}}\left(T_{m}\right)=0.31 \pm 0.03$ which coincides with the value of $\frac{1}{3}$ from the KTBHNY theory. The fact that our results agree with the KTBHNY pre- 


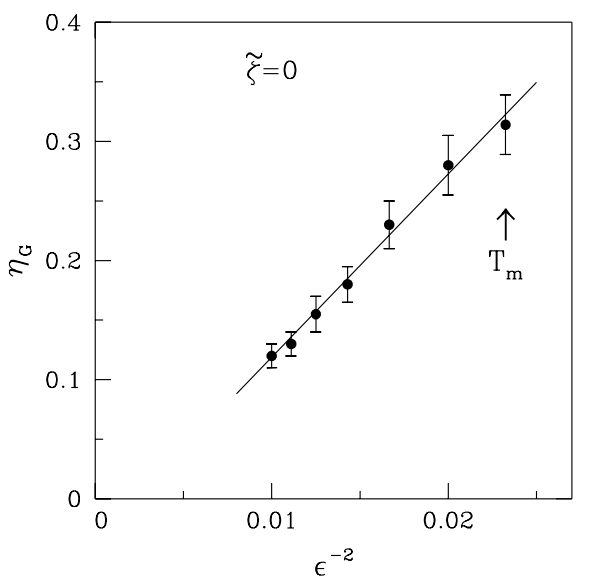

FIG. 6: Dependence of $\eta_{\mathbf{G}}(T)$ on $\epsilon^{-2}$ for the pure system. The arrow indicates the melting temperature which corresponds to $\epsilon_{c}^{2}=43$.

dictions suggests that the first order transition is weak and the second phase transition theory still applies. The weakness of the first order transition was supported by analytical calculations of Hikami et $a^{112}$ who obtained a small difference between free energies of the liquid and quasi-long range lattice phases at the melting temperature. Since in the LLL approximation screening effects are neglected, $\lambda \rightarrow \infty$ and hence, as follows from Eq. (2), $\eta_{\mathbf{G}}=\frac{G^{2} T}{4 \pi \mu} \sim \frac{1}{\epsilon^{2}}$. The results shown in Fig. [6]demonstrate that, in agreement with the theoretical prediction ${ }^{6}$, $\eta_{\mathbf{G}}(T) \sim \frac{1}{\epsilon^{2}}$.

\section{DISORDERED SYSTEM}

In this section we will study the effect of weak disorder on the flux lattice melting transition and on the behavior of different correlation functions. Fig. [7] shows a typical snapshots of vortex cores obtained for the clean case $(\tilde{\zeta}=$ $0)$ and two values of the disorder strength: $\tilde{\zeta}=0.03$ and 0.2 after $500000 \mathrm{MC}$ steps. The runs were done at $\epsilon^{2}=60$ for the system of $N_{\phi}=64$ vortices and are long enough so that the equilibrium was reached. For the uniform $(\tilde{\zeta}=0)$ and weakly disordered $(\tilde{\zeta}=0.03)$ cases we find the slightly distorted Abrikosov lattice. The dislocations are clearly seen in the case of $\tilde{\zeta}=0.2$ and the quasi-solid lattice phase cease to be exist. Thus, in the weak disorder strength limit dislocations can be neglected. We restrict ourselves to this limit and concentrate on two values of disorder $\tilde{\zeta}=0.01$ and 0.03 . Some modest simulations have been performed also for $\tilde{\zeta}=0.02$ and 0.04 .

The MC simulations were carried out for systems of $N_{\phi}=4^{2}, 6^{2}, 8^{2} 10^{2}$ and $12^{2}$ vortices. Depending on $N_{\phi}$, $10^{5}-2 \times 10^{6} \mathrm{MC}$ steps were used and half of them were spent on equilibration of the system. The disorder aver-
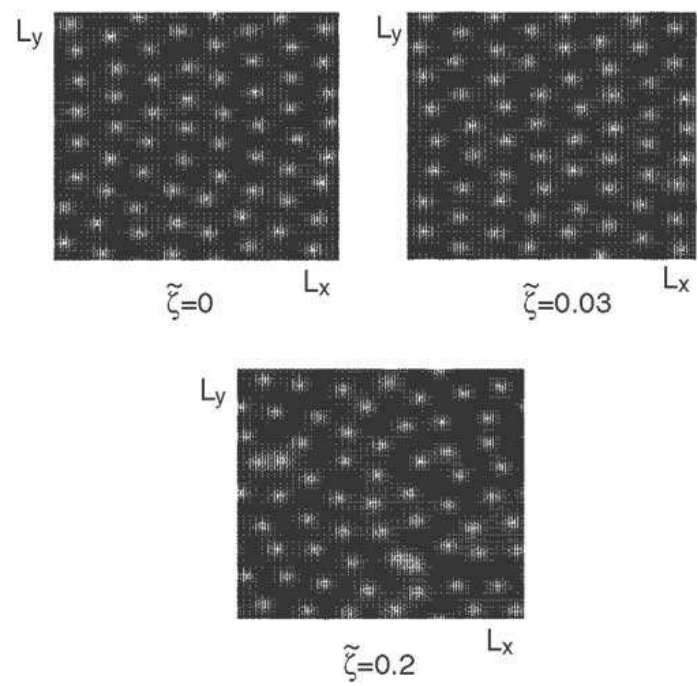

FIG. 7: Snapshots of the vortex positions for $\tilde{\zeta}=0,0.03$ and 0.2 obtained at $\epsilon^{2}=60$ after $500000 \mathrm{MC}$ steps. Light spots correspond to vortex cores. We choose $N_{\phi}=64$.

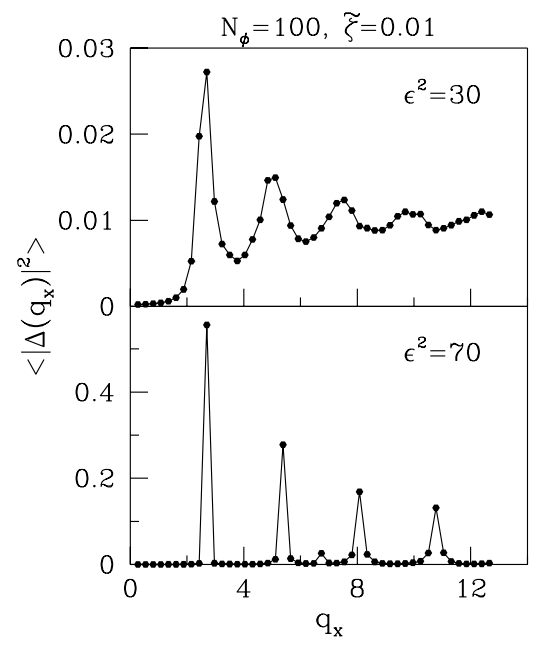

FIG. 8: The wave vector dependence of the structure factor for the disordered system with $\tilde{\zeta}=0.01 . N_{\phi}=100, \epsilon^{2}=30$ and 70 .

age is done typically over $10-40$ samples.

Fig. 8 shows the temperature dependence of the intensity of Bragg peaks defined by $\left\langle\left|\Delta\left(q_{x}\right)\right|^{2}>\right.$ for high $\left(\epsilon^{2}=30\right)$ and low $\left(\epsilon^{2}=70\right)$ temperatures, $\tilde{\zeta}=0.01$ and $N_{\phi}=100$. As in the clean case, at low $T$ 's we still have sharp peaks characterizing the ordering of the vortices.

The transition temperature to the vortex liquid phase may be defined from the finite size scaling analysis of the structure factor. Fig. 9 shows the temperature dependence of $\left\langle|\tilde{\Delta}(G)|^{2}>\right.$ given by Eq. (24) for various system sizes and $\tilde{\zeta}=0.01$. The curves splay out at $\epsilon_{c}^{2}=48 \pm 2$ indicating that the disorder reduces slightly the transition temperature to the liquid phase. For $\tilde{\zeta}=0.03$ we have $\epsilon_{c}^{2}=57 \pm 2$ (the results are not 
shown).

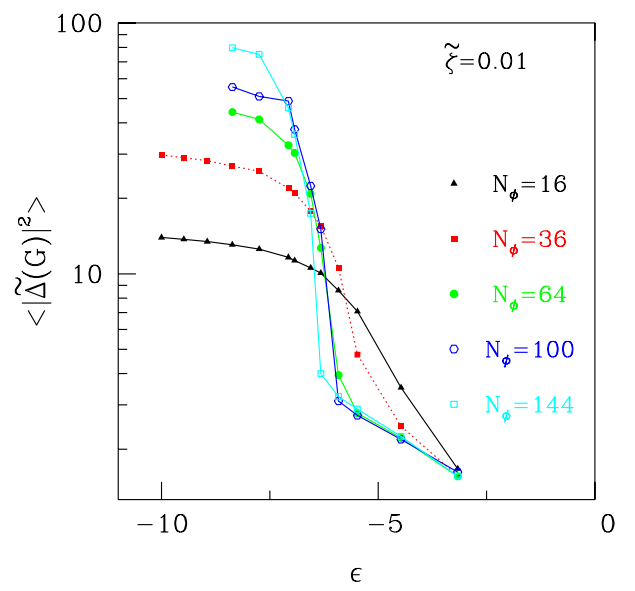

FIG. 9: The temperature dependence of the structure factor for the disordered system with $\tilde{\zeta}=0.01$. The curves splay out at $\epsilon_{c}^{2}=48 \pm 2$.

To calculate from the critical value $\epsilon_{c}\left(\tilde{\zeta}_{j}\right) \equiv \epsilon_{c, j}$ the true phase boundary $b_{c}(t, G i, \zeta)$ for the four given values of $\tilde{\zeta}_{j}\left(\tilde{\zeta}_{1}=0.01, \tilde{\zeta}_{2}=0.02, \tilde{\zeta}_{3}=0.03\right.$ and $\tilde{\zeta}_{4}=0.04$ ) we use the relation (21). Since the maximal value of $t$ is one, the maximum value of $\zeta$ is given by $\zeta_{j}(G i)=2 \sqrt{\pi} G i^{\frac{1}{4}} \tilde{\zeta}_{j} \epsilon_{c, j}$. Having chosen for a given Ginzburg number $G i$ the values of $\zeta$ smaller than $\zeta_{j}(G i)$, we find that the relation (21) defines a temperature $t_{j}=\frac{\zeta^{2}}{4 \pi G i^{1 / 2} \tilde{\zeta}_{j}^{2} \epsilon_{c j}^{2}}=\frac{\zeta^{2}}{\zeta_{c j}^{2} G i}$ from which we get $b_{c}\left(t_{j}, G i, \zeta\right)=\tilde{b}_{c}\left(t_{j}, \epsilon_{c j} G i^{\frac{1}{4}}\right)$. Here function $\tilde{b}_{c}$ is defined by Eq. (16).

Fig. 10] shows the $b-t$ phase diagram for the disordered case for $G i=0.01$. The true disorder strength is equal to $\zeta=0.06$. The dashed line corresponds to the clean system. Obviously, the fluctuations due to disorder shift the transition line to lower magnetic fields. The inset shows the dependence of the Ginzburg-Landau energy distribution function $P(E)$ on $E$ for $N_{\phi}=144$ and $\epsilon^{2}=$ 50 . The double peak structure gives strong evidence that the transition is first order.

The question we ask now is what is the critical disorder strength $\tilde{\zeta}_{c}$ above which the COCD glass phase disappear at any nonzero temperature. To answer this question one has to, in principle, find the dependence of the effective critical temperature $\epsilon_{c}^{2}$ on $\tilde{\zeta}$. But for large values of $\tilde{\zeta}$ it is very difficult to equilibrate the system at low temperatures and one cannot locate $\epsilon_{c}^{2}$. Therefore, we restrict ourselves to a few values of the disorder strength and plot $1 / \epsilon_{c}^{2}$ versus $\tilde{\zeta}$ as shown in Fig. [1] The interpolation to $1 / \epsilon_{c}^{2}=0$ which corresponds to the real zero temperature, gives $\tilde{\zeta}_{c} \approx 0.11$.

Fig. 12 shows the $\ln S(G, \mathbf{r})-\ln r$ plot for $S(\mathbf{G}, \mathbf{r})$ defined in (5) of the $N_{\phi}=12 \times 12$ disordered system with $\tilde{\zeta}=0.01$. For $\epsilon^{2}>\epsilon_{c}^{2}=48 \ln S(\mathbf{G}, \mathbf{r})$ decays linearly,

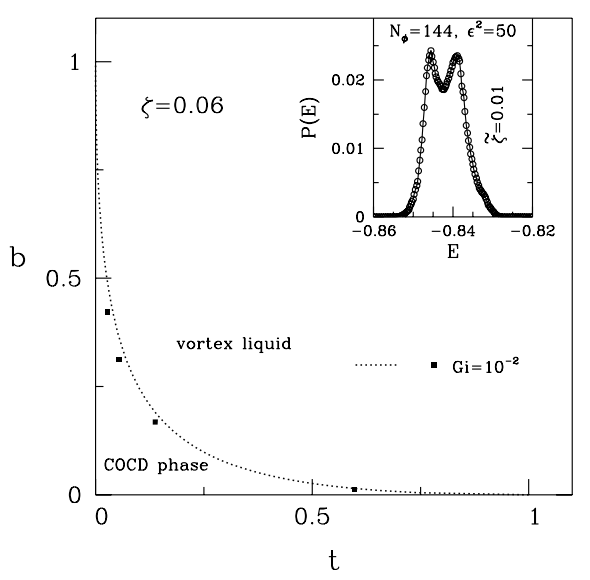

FIG. 10: The $t-b$ phase diagram for the disordered system with disorder strength $\zeta=0.06$. We chose $G i=10^{-2}$. The closed squares denote the position of the melting line in the presence of disorder and the dotted line represents the case without disorder. In the disordered case the glassy COCD phase exists at low temperatures. The inset shows the distribution $P(E)$ of the Ginzburg-Landau energy measured in units of the mean-field energy $E_{M F}=N_{\phi} k_{B} T \epsilon^{2} / \beta_{A}$, where $\beta_{A}$ is the mean field value of the Abrikosov ratio. $P(E)$ was obtained for $N_{\phi}=144$ and $\epsilon^{2}=50$

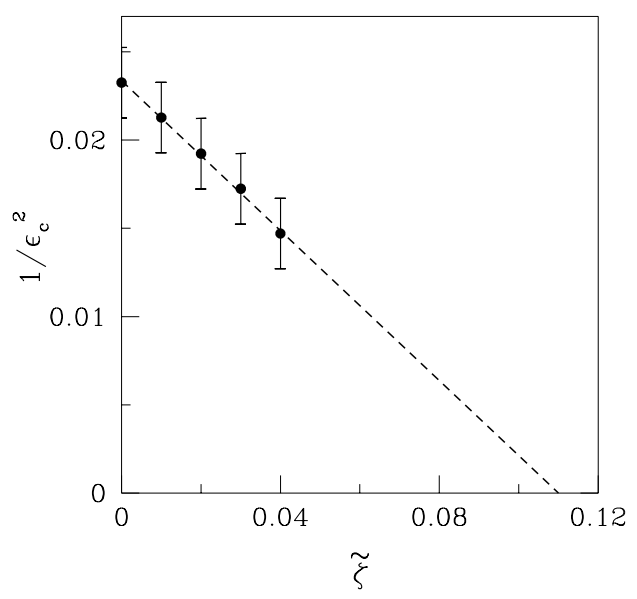

FIG. 11: The dependence of $\epsilon_{c}^{2}$ on $\tilde{\zeta}$. The interpolation by a straight line gives $\tilde{\zeta}_{c} \approx 0.11$.

except for $\epsilon^{2}=50$ where the decay is much faster. A slightly better fit can be obtained if one plots $\ln S(G, \mathbf{r})$ versus $\ln ^{2} r$ as shown in Fig. 13 In this case $S(\mathbf{G}, \mathbf{r}) \sim$ $\exp \left(-\tilde{\eta}_{\mathbf{G}} \ln ^{2} r\right)$, in agreement with the prediction of the COCD theory ${ }^{35.36}$. The exponent $\tilde{\eta}_{\mathbf{G}}(T)$ depends not only on $T$ but, as we will see below, also on the disorder strength .

Fig. 14 shows the dependence of the translational invariance order parameter $S(G, \mathbf{r})$ on $r$ for $\tilde{\zeta}=0.03$. In this case we still have the roughness $\sim \ln ^{2} r$ but the decay 


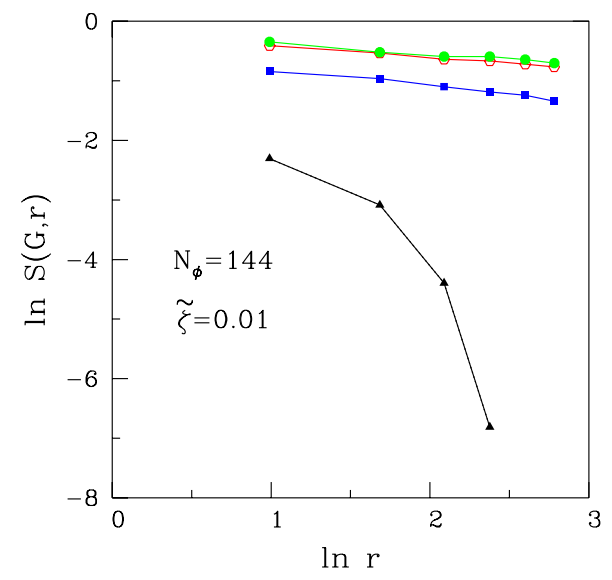

FIG. 12: The distance dependence of the translational invariance order parameter $S(G, \mathbf{r})$ for the disordered system with $N=144$ vortices. $\tilde{\zeta}=0.01$, closed triangles, squares, open hexagons and closed circles correspond to $\epsilon^{2}=30,50,60$ and 70 , respectively.

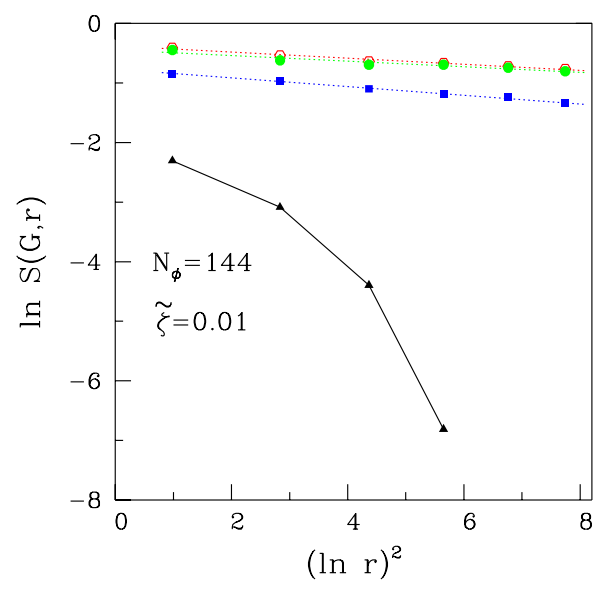

FIG. 13: The same as in Fig. 12 but data are plotted versus $\ln ^{2} r$.

is faster than in the $\tilde{\zeta}=0.01$ case.

Fig. 15 shows the temperature and disorder dependence of exponent $\tilde{\eta}_{\mathbf{G}}(T)$ for $N_{\phi}=144$. From the simulation we find that $\tilde{\eta}_{\mathbf{G}}$ increases with the disorder strength. The COCD theory, on the other hand, predicts a universal disorder independent value of $\tilde{\eta}_{\mathbf{G}}$, provided one is in the asymptotic region where one sees true fixed point behavior. We interpret our numerical result as a crossover effect: because of the relatively small system sizes one is probably not yet in the asymptotic region of the COCD theory $\left(L_{s} \lesssim L_{c o}\right)$ and hence an increase of the disorder will results in a faster decay of the correlation. The other observation is that $\tilde{\eta}_{\mathbf{G}}$ increases with increasing temperature. The same remains true for $\eta_{\mathbf{G}}$ if we fit $S(\mathbf{G}, \mathbf{r}) \sim e^{-\eta_{\mathbf{G}} \ln r}$ (compare Fig. 12). The result for

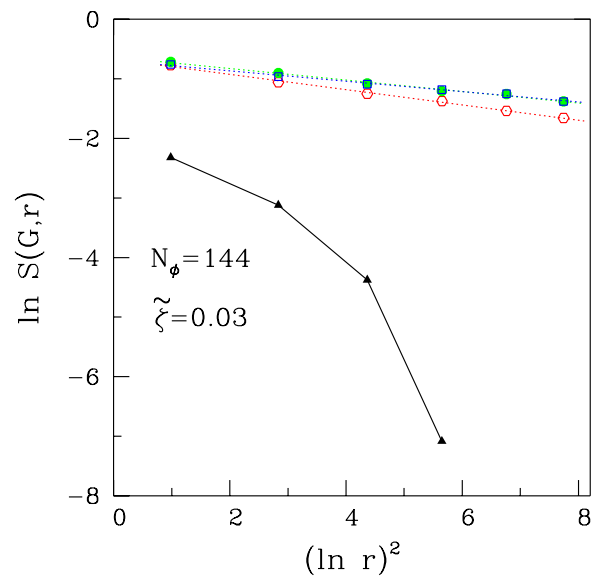

FIG. 14: The same as in Fig. 13] but for $\tilde{\zeta}=0.03$. Closed triangles, open hexagons, closed circles and open squares correspond to $\epsilon^{2}=30,60,70$ and 80 , respectively.

$\tilde{\eta}_{\mathbf{G}}$ is opposite to the expectation from the COCD theory in the asymptotic regime. However, our result can be understood again if we assume that the main effect for the roughness of the vortex lattice still comes from thermal fluctuations. They alone lead indeed to the increase of $\tilde{\eta}_{\mathbf{G}}$ with temperature. Thus, we interpret the result of Fig. [15] as a combined effect of thermal fluctuations and disorder on scales $L_{s} \lesssim L_{c o}$. The present data does not allow for a clear distinction between an $\ln r$ and $\ln ^{2} r$-behavior of $\ln S(\mathbf{G}, \mathbf{r})$.

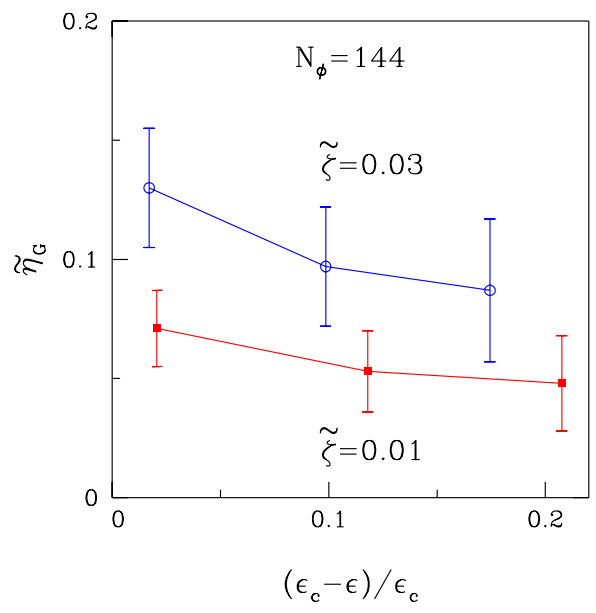

FIG. 15: The temperature and disorder dependence of $\tilde{\eta}_{\mathbf{G}}(T)$ characterizing the decay of the translational invariance correlation function in the super-rough phase. $N_{\phi}=144, \tilde{\zeta}=0.01$ (closed squares) and $\tilde{\zeta}=0.03$ (open hexagons). Error bars are mainly due to sample-to-sample fluctuations.

We next study the positional vortex glass ordering characterized by the order parameter $S_{P G}(\mathbf{G}, \mathbf{r})$ (6) 
which we try to fit as

$$
\ln S_{P G}(\mathbf{G}, \mathbf{r})=-\eta_{P G} \ln r+\text { const. }
$$

where $\eta_{P G}$ is an exponent. The COCD theory predicts an $\ln r$-behavior ${ }^{44}$ with $\eta_{P G} \sim T$. Fig. [16] shows the distance dependence of $S_{P G}(G, \mathbf{r})$ versus $\ln r$ for $\tilde{\zeta}=0.01$ and 0.03 . At low temperatures the fit given by Eq. (26) works well and the corresponding exponent $\eta_{P G}$ is presented in Fig. 17. As the temperature is lowered the tendency to the ordering gets more and more enhanced and $\eta_{P G}$ decreases. Fig. [17 shows that $\eta_{P G}$ is not sensitive to the disorder strength. Both findings are in agreement with the theoretical prediction ${ }^{44}$ suggesting a quasi-long range glass ordering of $e^{i \mathbf{G u}}$.

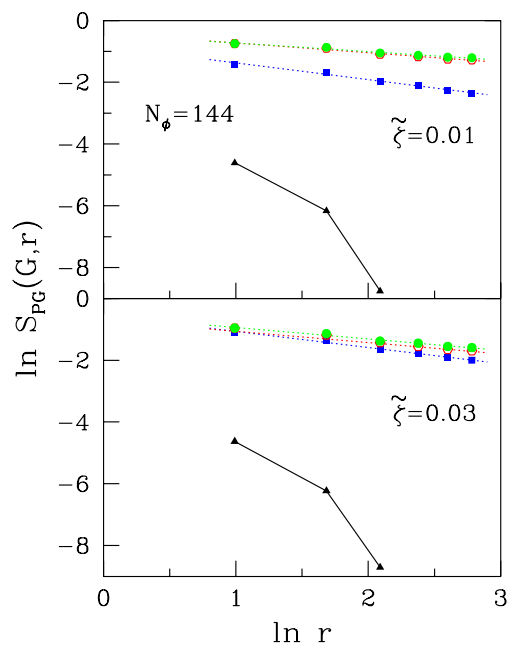

FIG. 16: $\operatorname{Ln} S_{P G}(G, \mathbf{r})-\ln r$ plot for $N_{\phi}=144, \tilde{\zeta}=0.01$ (upper pane) and $\tilde{\zeta}=0.03$ (lower panel). For $\tilde{\zeta}=0.01$ closed triangles, squares, open hexagons and closed circles correspond to $\epsilon^{2}=30,50,60$ and 70 , respectively. For $\tilde{\zeta}=0.03$ closed triangles, squares, open hexagons and closed circles correspond to $\epsilon^{2}=30,60,70$ and 80 , respectively.

Next we consider the vortex glass correlation function for a phase coherent vortex glass, Eq. (8). In order to compute the phase-sensitive correlation function $C_{V G}(\mathbf{r})$ we go to the symmetric gauge to obtain the phase of the order parameter. The typical spatial distribution of the phase in the symmetric gauge is shown in Fig. 18 It has essentially the same shape as presented in the work of Brandt 45 .

We fixed the order parameter at the center of the rectangular as shown in Fig. 18 as $\Psi(0)$ and compute $C_{V G}(\mathbf{r})$ with the help of Eq. (8), where $|\mathbf{r}|$ is the distance to this center. To improve statistics we average over 360 different directions of $\mathbf{r}$ keeping $|\mathbf{r}|=r$ fixed.

Fig. 19] and 20] show the spatial behavior of the vortex glass order parameter $C_{V G}(r)$ given by Eq. (8) for $\tilde{\zeta}=$ 0.01 and 0.03 . In the low temperature region straight lines correspond to the fit

$$
C_{V G}(\mathbf{r}) \sim \exp \left(-r / R_{c}\right)
$$

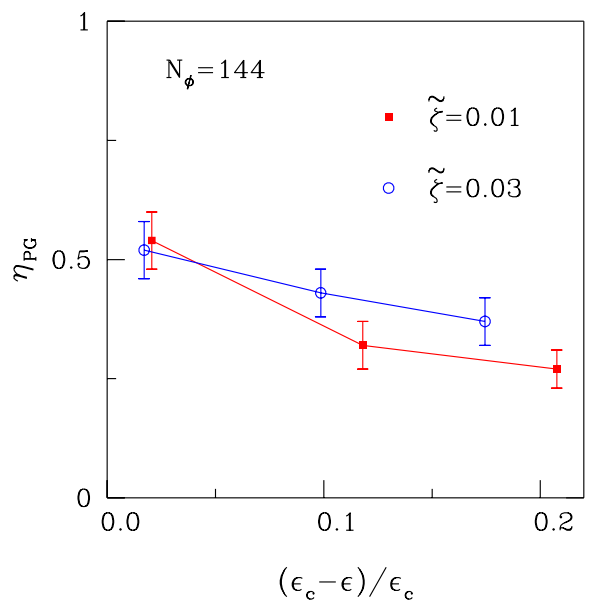

FIG. 17: Temperature dependence of $\eta_{P G}$ for $\tilde{\zeta}=0.01$ (closed squares) and 0.03 (open circles).

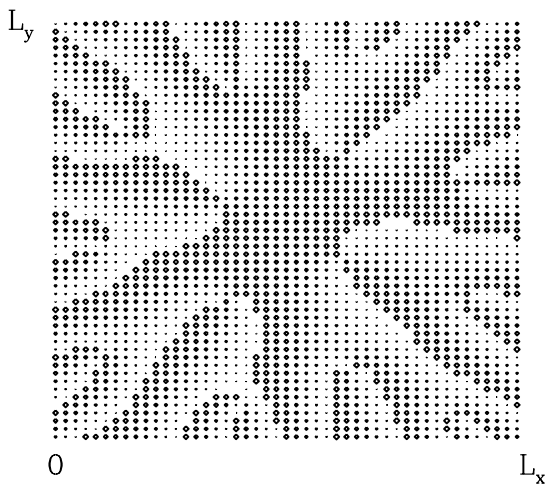

FIG. 18: Space distribution of the phase of the order parameter in the symmetric gauge. The darker region the larger is the value of the phase. The snapshot was obtained for $N_{\phi}=16$ after $3 \times 10^{5} \mathrm{MC}$ steps at $\epsilon^{2}=50$. The boundary between the dark and the bright region corresponds to the phase jump from $\varphi=2 \pi$ to $\varphi=0$. Going around the system the total phase change is $2 \pi N_{\phi}$.

where $R_{c}$ is the correlation length. At high temperatures $C_{V G}(\mathbf{r})$ decays faster than exponential.

Fig. 21 shows the temperature dependence of $R_{c}$. As the temperature decreases, the system gets more ordered and $R_{c}$ increases. The same tendency shows up if one increases the disorder strength. However we do not see any sign of a true or quasi-long range order of the phase coherent vortex glass correlation function. 


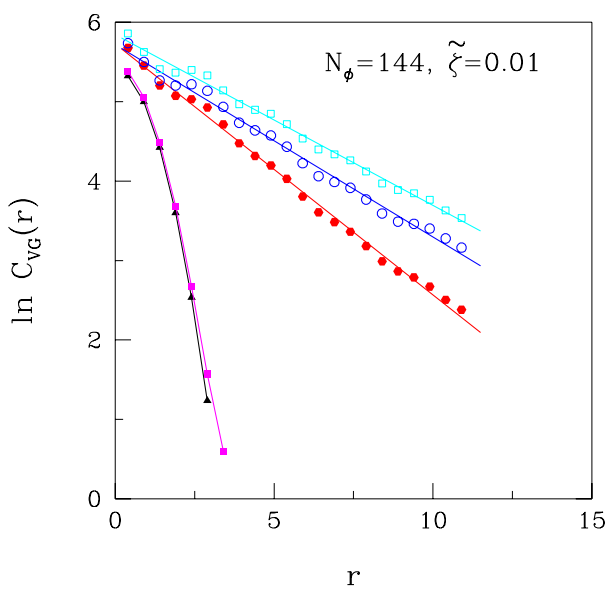

FIG. 19: The spatial behavior of the vortex glass order parameter $C_{V G}(\mathbf{r})$ in the $\ln C_{V G}(\mathbf{r})-r$ plot. The closed triangles, squares, hexagons, open circles and squares correspond to $\epsilon^{2}=10,30,50,60$ and 70 , respectively. Straight lines are linear fits $\log C_{V G}(R) \sim R$ for $\epsilon^{2}=50,60$ and $70 . \quad N=144$ and $\tilde{\zeta}=0.01$.

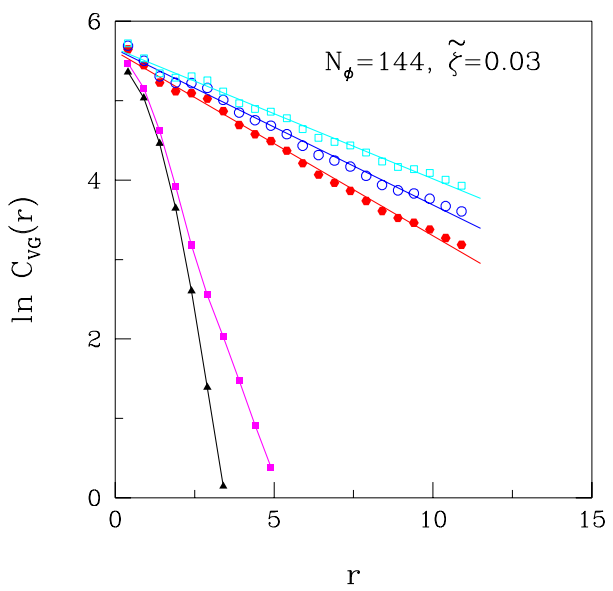

FIG. 20: The same as in Fig. [19] but for $\tilde{\zeta}=0.03$. The closed triangles, squares, hexagons, open circles and squares correspond to $\epsilon^{2}=10,30,60,70$ and 80 , respectively. The straight lines are fits for $\epsilon^{2}=60,70$ and 80 .

\section{CONCLUSION}

We have studied the phase diagram and different correlation functions in a $2 \mathrm{D}$ type II superconductor by Monte Carlo simulations using the LLL approximation. For the clean case, in accord with the previous results of other groups ${ }^{12.13 .18 .19}$, we have shown that, the quasi- long range ordered vortex lattice phase exists at low temperatures. The exponent $\eta_{\mathbf{G}}$ was found to be proportional to $\epsilon^{-2}$ as expected from the KTBHNY theory of the dislocation driven melting transition. This result suggests that the transition from the quasi-solid phase to the

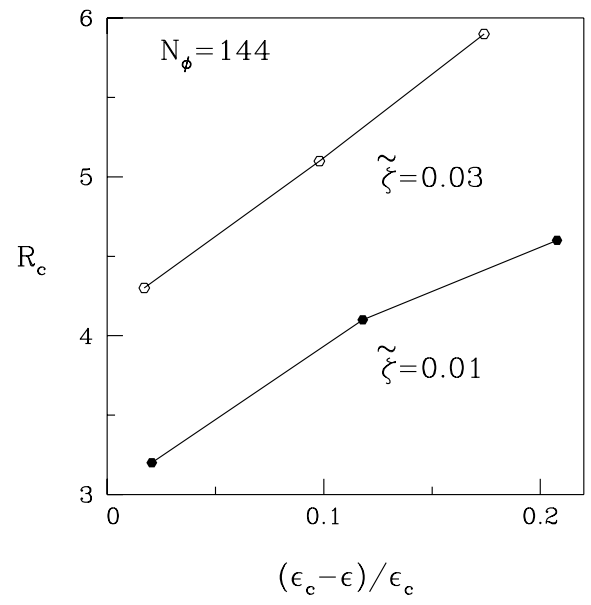

FIG. 21: Temperature dependence of $R_{c}$ for the phase coherent vortex glass order parameter in the COCD glass phase. $N_{\phi}=144, \tilde{\zeta}=0.01$ and $\tilde{\zeta}=0.03$.

liquid one is weakly first order. It is not excluded that the agreement between our results and the predictions of the KTBHNY theory is just an artifact of the finite size effect.

In disordered systems there is a first order transition from vortex liquid phase to a COCD phase characterized by the $\ln ^{2} r$-behavior of the roughness. This phase is however only stable for small enough disorder strength, $\tilde{\zeta} \lesssim \tilde{\zeta}_{c} \approx 0.11$. In the COCD phase the positional vortex glass correlation function decays as power law. In agreement with the COCD theory, the corresponding exponent $\eta_{P G}$ grows with $T$ linearly and remains almost unaffected by the disorder.

The phase coherent vortex glass correlation function decays with $r$ exponentially (see Eq. (27)) where the correlation length $R_{c}$ increases with disorder indicating that the disorder favours the vortex glass ordering. However the phase coherent vortex glass is suppressed by thermal fluctuations in weakly disordered 2D systems.

We thank Y. Kato and A. Glatz for many useful discussions and D. Stauffer for his kind help in implementation of the code for parallel computation on CRAY located at the John von Neumann Institute for Computing. The financial support from SFB 608 and the Polish Agency KBN (Grant number 2P03B-146-18) is acknowledged.
1 A. A. Abrikosov, Sov. Phys. JETP 5, 1174 (1957).

${ }^{2}$ G. Eilenberger, Phys. Rev. 164, 628 (1967).
3 J. M. Kosterlitz and D. J. Thouless, J. Phys. C 6, 1181 (1973). 
${ }^{4}$ V.L. Berezinskii, Sov. Phys. JETP 32, 493 (1970), 34, 610 (1971).

5 B. I. Halperin and D. R. Nelson, Phys. Rev. Lett. 41, 121 (1978).

6 D. R. Nelson and B. I. Halperin, Phys. Rev. B 19, 2457 (1979)

7 A. P. Young, Phys. Rev. B 19, 1855 (1979).

8 S. Doniach and B. A. Huberman, Phys. Rev. Lett. 42, 1169 (1979).

9 D. S. Fisher, Phys. Rev. B 22, 1190 (1980).

10 C. E Creffield and J. P. Rodriguez, cond-mat/0205231

11 P. Gupta and S. Teitel, Phys. Rev. 55, 2756 (1997) and references therein.

12 S. Hikami, A. Fujita and A. I. Larkin, Phys. Rev. B 44, 10400 (1991).

13 Z. Tesanovic and L. Xing, Phys. Rev. Lett. 67, 2729 (1991)

14 D. J. Thouless, Phys. Rev. Lett. 34, 946 (1975); G. J. Ruggeri and D. J. Thouless, J. Phys. F 6, 2063 (1976).

15 D. Yoshioka, B. I. Halperin, and P. A. Lee, Phys. Rev. Lett. 50, 1219 (1983).

16 M. A. Moore, Phys. Rev. B 45, 7336 (1992)

17 J. Yeo and M. A. Mooore, Phys. Rev. Lett. 76, 1142 (1996); Phys. Rev. B 54, 4218 (1996).

18 J. Hu and A. H. MacDonald, Phys. Rev. Lett. 71, 432 (1993).

19 Y. Kato and N. Nagaosa, Phys. Rev. B 47, 2932 (1993); Phys. Rev. B 48, 7383 (1993).

20 J. A. O'Neill and M. A. Moore, Phys. Rev. B 48, 374 (1993)

21 H. H. Lee and M. A. Moore, Phys. Rev. B 49, 9240 (1994)

${ }^{22}$ M. J. W. Dodgson and M. A. Moore, Phys. Rev. B 55, 3816 (1997).

23 A. I. Larkin, Sov. Phys. JETP 31, 784 (1970).

24 A. I. Larkin and Yu. N. Ovchinnikov, J. Low. Temp. Phys. 34, 409 (1979).

25 T. Nattermann, Phys. Rev. Lett. 64, 2454 (1990)
26 S. E. Korshunov, Phys. Rev. B 48, 3969 (1993)

27 T. Giamarchi and P. Le Doussal, Phys. Rev. Lett. 72, 1530 (1994); Phys. Rev. B 52, 1242 (1995).

28 T. Emig, S. Bogner and T. Nattermann, Phys. Rev. Lett. 83, 400 (1999); S. Bogner, T. Emig and T. Nattermann, Phys. Rev. B 63, 174501 (2001).

29 J. Kierfeld, T. Nattermann and T. Hwa, Phys. Rev. B 55, 626 (1997)

30 D. Carpentier, P. Le Doussal and T. Giamarchi, Europhys. Lett. 35, 379 (1996)

31 D. S. Fisher, Phys. Rev. Lett. 78, 1964 (1997)

32 M. J. P. Gingras and D. A. Huse, Phys. Rev. B 53, 15193 (1996)

33 A. van Otterlo, R. T. Scalettar and G. T. Zimanyi, Phys. Rev. Lett. 81, 1497 (1998).

34 T. Klein, J. Joumard, S. Blanchard, J. Marcus, R. Cubitt, T. Giamarchi and P. Le Doussal, Nature 413, 404 (2001).

35 D. Carpentier and P. Le Doussal, Phys. Rev. B 55, 12128 (1997).

36 J. L. Cardy and S. Ostlund, Phys. Rev. B 25, 6899 (1982).

37 T. Nattermann and S. Scheidl, Adv. Phys. 49, 607 (2000).

38 P. Le Doussal and T. Giamarchi, Physica C 331233 (2000).

39 A. K. Kienappel and M. A. Moore, Phys. Rev. B 56, 8313 (1997).

40 K. Binder and A. P. Young, Rev. Mod. Phys. 58, 801 (1986).

41 D. S. Fisher, M. P. A. Fisher and D. Huse, Phys. Rev. B 43, 130 (1991).

42 D. Li and B. Rosenstein, Phys. Rev. B 60, 9704 (1999).

43 C. J. Lobb, Phys. Rev. B 36, 3930 (1987).

44 Y. Y. Goldschmidt and A. Houghton, Nucl. Phys. B 210, 155 (1982)

45 E. H. Brandt, Phys. Status Solidi 64, 257 (1974). 